\title{
THE METHOD OF EQUAL PROPORTIONS
}

\author{
LAURENCE F. SCHMECKEBIER*
}

The Constitution provides that the members of the House of Representatives shall be apportioned among the states according to their respective members. Not only the representation of the several states is concerned, but an inequitable apportionment has an effect on the vote in the electoral college, as the vote of any state is equal to the whole number of senators and representatives to which a state may be entitled. It is thus apparent that an equitable assignment of representatives to the several states is of paramount importance, as in a close election the result may be decided by the electoral votes of a state or group of states which had representation out of proportion to the population.

At first glance it might seem that with a given membership the population of each district would be determined by dividing the total population by the total number of members. Thus if the total population is $150,000,000$, and the membership of the House is fixed at 435 , there should be one member for each 344,827 population, and the apportionment of each state would be its population divided by 344,827 . But no state is likely to have a population equal to an exact multiple of the apportionment base. Fractions of various size would be left over, and it is the determination of the weight to be given to these fractions that constitutes the problem of an equitable apportionment.

\section{I}

\section{Factors Affecting the Several Methods}

Several factors bearing on the choice of a method should be borne in mind. The first of these is the average population per district which is the result of dividing the population of a state by the number of representatives. It is consequently the same as the average population per representative.

Another factor of importance is the share of the individual citizen in a representative. This is obtained by dividing the number of representatives from the state by the total population. Thus if the population of a state is 687,497 , and there are two representatives, the share of each individual is 2 divided by 687,497 , or $.000,002,9$ or 2.9 per million.

In valuating the differences in the average population and in the individual share in a representative, consideration must be given to the absolute and relative differences. The absolute difference is obtained by substracting the smaller from the larger number. The relative difference is the percentage by which the larger exceeds

A.B. 1896 , Ph.D. I899, Johns Hopkins University. Retired. Staff member of the Institute for Government Research of the Brookings Institution, 1921-1942; chairman, 1941-1942. Author, CoNGREsstonAI. APFORTIONMENT (I94I), and various books on government organization and activitics. 
the smaller. Thus if a piece of property costing $\$ 500$ is sold for $\$ 600$ and another parcel costing $\$ 1,000$ is sold for $\$ I, I 00$ the absolute difference in the profit is the same in each case-namely, \$100. But the relative difference in one case is 500 divided into I00, or 20 per cent; in the other case it is 1,000 divided into roo or ro per cent.

All modern methods of apportionment are based on priority lists, which indicate the apportionment of each state for any size of the House. As the Constitution provides that one representative must be assigned to each state, a priority list begins with the forty-ninth member of the House or the second member from some state.

The priority list numbers are obtained by multiplying the population of each state successively by the numbers applicable to the second member, the third member, and so on. As the several methods are based on different formulae, a different series of multipliers is used for each method. The formulae for obtaining the priority numbers are given under the discussion of each method.

Having obtained the priority numbers as described above, those for each method are arranged in descending order. The priority list for the method of equal proportions, based on the census of I950, would then read in part as follows:

Section of Priority List for Method of Equal Proportions, Based on Census of ig5o

\begin{tabular}{|c|c|c|c|}
\hline $\begin{array}{l}\text { Total number of } \\
\text { representatives } \\
\text { from all states }\end{array}$ & $\begin{array}{c}\text { Priority list } \\
\text { number }\end{array}$ & State & $\begin{array}{l}\text { Cumulative total } \\
\text { of representatives } \\
\text { from each state }\end{array}$ \\
\hline $\begin{array}{l}49 \\
50 \\
51 \\
52\end{array}$ & $\begin{array}{r}10,486,529 \\
7,485,590 \\
7,423,215 \\
6,160,438\end{array}$ & 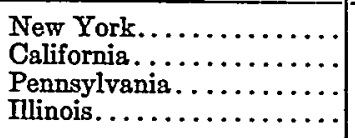 & $\begin{array}{l}2 \\
2 \\
2 \\
2\end{array}$ \\
\hline 100 & $1,658,275$ & North Carolina........... & 3 \\
\hline 200 & 770,231 & Georgia............. & 5 \\
\hline 300 & 507,923 & Tennessee.............. & 7 \\
\hline 400 & 377,059 & New Hampshire....... & 2 \\
\hline $\begin{array}{l}433 \\
434 \\
435\end{array}$ & $\begin{array}{l}348,627 \\
347,858 \\
347,684\end{array}$ & 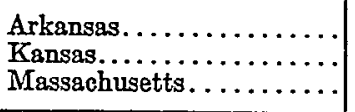 & $\begin{array}{r}6 \\
6 \\
14\end{array}$ \\
\hline $\begin{array}{l}436 \\
437\end{array}$ & $\begin{array}{l}347,135 \\
347,048\end{array}$ & 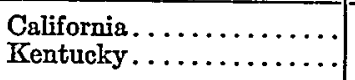 & $\begin{array}{r}31 \\
9\end{array}$ \\
\hline
\end{tabular}

A line is then drawn below the number in the first column representing the membership that has been determined upon. It should be noted that the first number is 49 , as one representative has already been assigned by constitutional provision to each state. Thus for each state the last entry above the line in the fourth column (cumulative total of representatives from each state) shows the number of representatives to which each state is entitled. Thus for a house of 435 members, reading up from 435 , the members apportioned to the last 3 states would 
be Arkansas 6, Kansas 6, and Massachusetts 14. If the membership were increased to 436 , California would obtain an additional member; if the membership were decreased to 434 , Massachusetts would be reduced to 13 .

II

Advantages of Methods of Equal Proportions

By the method of equal proportions the difference between the representation of any two states is the smallest possible when measured by the relative difference in the average population per district and also by the relative difference in the individual share in a representative. These features appear to make it superior to any other method that has been devised.

Under this method state $A$ deserves an additional representative when its population divided by the geometric mean of its present assignment of representatives and of its next higher assignment is greater than the population of any other state divided by the geometric mean of the assignment to such other state and its next higher assignment. The geometric mean of any two numbers is the square root of their product; the geometric mean (m) of two numbers (a) and (b) may be expressed as follows:

$$
\frac{m}{a}=\frac{b}{m} \text { or } m^{2}=a b, \text { or } m=\sqrt{a b}
$$

Thus the geometric mean of 4 and 16 is

$$
\frac{\mathrm{m}}{4}=\frac{16}{\mathrm{~m}} \text { or } \mathrm{m}^{2}=16 \times 4 \text { or } \mathrm{m}=\sqrt{64=8}
$$

In order to compute the relative claim of each state a priority list is prepared as heretofore described; the process for the method of equal proportions is expressed in the form of an equation as follows:

$P$ (priority Population of any state (A)

list figure) $=\overline{\text { Geometric mean of its next higher assignment }(\mathrm{k}) \text { and its present }}$ assignment $(k-1)$

As one representative has already been assigned, the priority number for the second member will be as follows:

$$
\begin{aligned}
\mathrm{P} 2 & =\frac{A}{\sqrt{\mathrm{k}(\mathrm{k}-1)}} \\
& =\frac{A}{\sqrt{2(1)}} \\
& =\frac{A}{\sqrt{2}} \\
& =\frac{A}{1.414,213,6} \\
& =\mathrm{A} \times \text { reciprocal of } 1.414,213,6 \\
& =\mathrm{A} \times 0.707,106,78
\end{aligned}
$$


The last figure in the above equation $(0.707,106,78)$ is then multiplied by the population of the state in order to obtain the priority number for the second member. For each succeeding priority list number, $\mathrm{k}$ is increased by one; thus the formulae for the third and fourth members will be as follows:

$$
\begin{aligned}
& \mathrm{P} 3=\frac{A}{\sqrt{3(3-1)}} \\
& \mathrm{P} 4=\frac{A}{\sqrt{4(4-1)}}
\end{aligned}
$$

The multipliers to be applied to the population in order to obtain the priority list for the method of equal proportions are given on page 24 of my book, Congressional Apportionment. ${ }^{1}$

The superiority of the method of equal proportions may be tested arithmetically by a comparison of the representation of any two states by the method of equal proportions and by any other method. If any two states are compared, it will be found that the relative difference in the average population per district is less by the method of equal proportions than by any other method. Likewise, if the relative difference in the individual share in a representative is compared, it will be found that the difference is smaller in the case of the method of equal proportions.

The algebraic proof of the superiority of the method of equal proportions is shown in the following computation:

The first problem is to find a method of assignment of representatives among the states so that the relative difference in average population of districts between any two states is as small as possible.

Designate the population of two states as $A$ and $B$; designate the number of representatives assigned to State $A$ as $a$, and the number of representatives assigned to State $B$ as $b$. The average population of the districts in any state is the total population divided by the number of representatives, or $\frac{A}{a}$ and $\frac{B}{b}$.

Under what conditions would it be fairer to assign an additional representative to State $A$ in preference to State $B$ ? If an additional representative is assigned to $A$ the relative difference in average population per district would be expressed by the following fraction:

$$
\frac{\left(\frac{B}{b}\right)-\left(\frac{A}{a+1}\right)}{\left(\frac{A}{a+1}\right)} .
$$

If an additional representative is assigned to $B$ the relative difference in average population per district would be expressed by the following fraction:

\footnotetext{
1 Laurence F. Schmeckebier, Congressional Apportionment (The Brookings Institution, $194 \mathrm{r}$ ).
} 


$$
\frac{\left(\frac{A}{a}\right)-\left(\frac{B}{b+1}\right)}{\left(\frac{B}{b+1}\right)}
$$

If the assignment of the additional representative to $A$ is correct, the relative difference will be smaller than if the additional representative had been assigned to $B$. This may be expressed as follows:

By separating the fractions we get

$$
\frac{\left(\frac{B}{b}\right)-\left(\frac{A}{a+1}\right)}{\left(\frac{A}{a+1}\right)}<\frac{\left(\frac{A}{a}\right)-\left(\frac{B}{b+1}\right)}{\left(\frac{B}{b+1}\right)} .
$$

$$
\frac{\left(\frac{B}{b}\right)}{\left(\frac{A}{a+1}\right)}-\frac{\left(\frac{A}{a+1}\right)}{\left(\frac{A}{a+1}\right)}<\frac{\left(\frac{A}{a}\right)}{\left(\frac{B}{b+1}\right)}-\frac{\left(\frac{B}{b+1}\right)}{\left(\frac{B}{b+1}\right)}
$$

As any number divided by itself is I we can dispense with the fractions

$$
\frac{\left(\frac{A}{a+1}\right)}{\left(\frac{A}{a+1}\right)} \text { and } \frac{\left(\frac{B}{b+1}\right)}{\left(\frac{B}{b+1}\right)}
$$

and write

$$
\frac{\left(\frac{B}{b}\right)}{\left(\frac{A}{a+1}\right)}-1<\frac{\left(\frac{A}{a}\right)}{\left(\frac{B}{b+1}\right)}-1
$$

We can add $\mathrm{r}$ to each side without changing the relationship and the result will be

$$
\frac{\left(\frac{B}{b}\right)}{\left(\frac{A}{a+1}\right)}<\frac{\left(\frac{A}{a}\right)}{\left(\frac{B}{b+1}\right)}
$$

If we multiply each side by both denominators the relationship will not be changed and we obtain the following:

$$
\begin{aligned}
& \frac{\left(\frac{B}{b}\right)}{\left(\frac{A}{a+1}\right)} \times\left(\frac{A}{a+1}\right) \times\left(\frac{B}{b+1}\right)<\frac{\left(\frac{A}{a}\right)}{\left(\frac{B}{b+1}\right)} \times\left(\frac{A}{a+1}\right) \times\left(\frac{B}{b+1}\right) \\
& \left(\frac{B}{b}\right) \times\left(\frac{B}{b+1}\right)<\left(\frac{A}{a}\right) \times\left(\frac{A}{a+1}\right) \\
& \frac{B^{2}}{b(b+1)}<\frac{A^{2}}{a(a+1)} \text {. }
\end{aligned}
$$


We can take the square root of both sides without changing the relationship, and we get

$$
\frac{B}{\sqrt{b(b+1)}}<\frac{A}{\sqrt{a(a+1)}} .
$$

For convenience we can turn this around to read

$$
\frac{A}{\sqrt{a(a+1)}}>\frac{B}{\sqrt{b(b+1)}} \text {. }
$$

As the square root of the product of two numbers is the geometric mean of these two numbers it follows that State $A$ deserves the additional representative when its population divided by the geometric mean of its present assignment and of its next higher assignment is greater than the population of State $B$ divided by the geometric mean of $B$ 's present assignment and of its next higher assignment.

The second problem is to find a method of assignment of representatives among the states so that the relative difference in the share in a representative between any two states is as small as possible.

If the population of two states is designated by $A$ and $B$ and the number of representatives by $a$ and $b$ as before, the share in a representative is the number of representatives divided by the population, or $\frac{a}{A}$ and $\frac{b}{B}$. If an additional representative is assigned to $A$ the relative difference per share of a representative would be

$$
\frac{\left(\frac{a+1}{A}\right)-\left(\frac{b}{B}\right)}{\left(\frac{b}{B}\right)}
$$

If an additional representative is assigned to $B$ the relative difference in the share of a representative would be expressed by the following fraction:

$$
\frac{\left(\frac{b+1}{B}\right)-\left(\frac{a}{A}\right)}{\left(\frac{a}{A}\right)}
$$

If the assignment of an additional respresentative to $A$ is correct the relative difference will be smaller than if the additional representative had been assigned to $B$. This may be expressed as follows:

$$
\frac{\left(\frac{a+1}{A}\right)-\left(\frac{b}{B}\right)}{\left(\frac{b}{B}\right)}<\frac{\left(\frac{b+1}{B}\right)-\left(\frac{a}{A}\right)}{\left(\frac{a}{A}\right)} .
$$


By separating the fractions we get:

$$
\frac{\left(\frac{a+1}{A}\right)}{\left(\frac{b}{\bar{B}}\right)}-\frac{\left(\frac{b}{B}\right)}{\left(\frac{b}{B}\right)}<\frac{\left(\frac{b+1}{B}\right)}{\left(\frac{a}{A 1}\right)}-\frac{\left(\frac{a}{A}\right)}{\left(\frac{a}{A}\right)} .
$$

As any number divided by itself is I we can dispense with the fractions and write

$$
\frac{\left(\frac{a+1}{A}\right)}{\left(\frac{b}{B}\right)}-1<\frac{\left(\frac{b+1}{B}\right)}{\left(\frac{a}{A}\right)}-1 .
$$

We can add I to each side without changing the relationship, and the result will be:

$$
\frac{\left(\frac{a+1}{A}\right)}{\left(\frac{b}{B}\right)}<\frac{\left(\frac{b+1}{B}\right)}{\left(\frac{a}{A}\right)}
$$

If we multiply each side by both denominators the relationship will not be changed, and we obtain the following

$$
\frac{\left(\frac{a+1}{A}\right)}{\left(\frac{b}{B}\right)} \times \frac{b}{B} \times \frac{a}{A}<\frac{\left(\frac{b+1}{B}\right)}{\left(\frac{a}{A}\right)} \times \frac{b}{B} \times \frac{a}{A}
$$

or

$$
\frac{a+1}{A} \times \frac{a}{A}<\frac{b+1}{B} \times \frac{b}{B}
$$

or

$$
\frac{a(a+1)}{A^{2}}<\frac{b(b+1)}{B^{2}}
$$

We can take the square root of both sides without changing the relationship and we get

$$
\frac{\sqrt{a(a+1)}}{A}<\frac{\sqrt{b(b+1)}}{B}
$$

By taking the reciprocals we get

$$
\frac{A}{\sqrt{a(a+1)}}>\frac{B}{\sqrt{b(b+1)}}
$$

As the square root of the product of two numbers is the geometric mean of these two numbers, it follows that State $A$ deserves the additional representative when its population divided by the geometric mean of its present assignment and its next higher assignment is greater than the population of State $B$ divided by the geometric mean of $B$ 's present assignment and its next higher assignment. 
The relative merits of the several methods were summed up as follows in I929 in a report of a Committee of the National Academy of Sciences: ${ }^{2}$

There are five methods of apportionment now known which are unambiguous (that is, lead to a workable solution), and should be considered at this time.

These five methods are-

Method of smallest divisors

Method of the harmonic mean

Method of equal proportions

Method of major fractions

Method of greatest divisors

After full consideration of these various methods your committee is of the opinion that on mathematical grounds, the method of equal proportions is the method to be preferred. Each of the other four methods listed is, however, consistent with itself and unambiguous.

Let the population of a State $A$ and the number of Representatives assigned to it according to a selected method of apportionment be a, and let $B$ and $b$ represent the corresponding numbers for a second State. Under an ideal apportionment the population $\mathrm{A} / \mathrm{a}, \mathrm{B} / \mathrm{b}$ of the congressional districts in the two States should be equal, as well as the numbers a/A, b/B of Representatives per person in each State. In practice it is impossible to bring this desirable result about for all pairs of States.

In the opinion of this committee the best test of a desirable apportionment so far proposed is the following:

An apportionment of representatives to the various States, when the total number of Representatives is fixed, is mathematically satisfactory if for every pair of States the discrepancy between the numbers $A / a$ and $B / b$ cannot be decreased by assigning one more Representative to the State $A$ and one fewer to the State $B$ or vice versa, or if the two numbers $a / A$ and $b / B$ have the same property.

The method of equal proportions is preferred by the committee because it satisfies the test proposed when applied either to sizes [population] of congressional districts or to numbers of representatives per person, and because it occupies mathematically a neutral position with respect to emphasis on larger and smaller States.

The following is a portion of the report on the methods of apportionment made in rg2I by the Census Advisory Committee of the Department of Commerce. ${ }^{3}$

The "method of major fractions" utilizes only one of several ways of expressing apportionment ratios. The "method of equal proportions" utilizes all of these ways without inconsistency. The latter method, therefore, has a broader basis.

In the "method of major fractions" the "nearness" of the ratios of representatives and population for the several States is measured by absolute differences. The "method of equal proportions" utilizes relative differences. The relative scale is to be preferred.

${ }^{2}$ Hearings before the House Committee on the Census on the Apportionment of Representatives in Congress, 76th Cong., $3 \mathrm{~d}$ Sess. 70-7 I (I940). The committee consisted of Raymond Pearl, chairman, G. A. Bliss, E. W. Brown, and L. P. Eisenhart.

${ }^{3}$ Hearings before the House Committee on the Census on H. R. 13471 (Apportionment of Representatives in Congress Amongst the Several States), pt. 2, 69th Cong., 2d Sess. 58 (1927). The committee consisted of W. S. Rossiter, chairman, C. W. Doten, E. F. Gay, W. C. Mitchell, E. R. A. Seligman, and A. A. Young. 
In I950 a committee on apportionment of the American Political Science Association recommended (one member dissenting) that "there is no reason for changing at this time from the mathematical formula in the present Apportionment Act of 194I, namely, the method of equal proportions."

\section{III}

\section{Other Methods of Apportionment}

By the method of major fractions the difference between the representation ot any two states is the smallest when measured by the absolute difference in the individual share of a representative. The priority list is computed by dividing the population of each state successively by the arithmetic mean between succeeding representatives. The equation for obtaining the multiplier would be as follows:

$$
\begin{aligned}
& \mathrm{P} \text { (priority } \\
& \text { list figure) }
\end{aligned}=\frac{\text { Population of any state (A) }}{\text { Its next higher assignment (k) minus } 1 \text { plus } 1 / 2}
$$

As one representative has already been assigned to each state, the formula for the second member would be as follows:

$$
\begin{aligned}
\mathrm{P} 2 & =\frac{\mathrm{A}}{(2-1)+1 / 2} \\
& =\frac{\mathrm{A}}{11 / 2} \\
& =\mathrm{A} \times \text { reciprocal of } 1.5 \\
& =\mathrm{A} \times 0.666,666,67
\end{aligned}
$$

The priority list number for the second member will then be the population multiplied by $0.666,666,67$. For each succeeding priority list number, $k$ is increased by one. Thus the formulae for the third and fourth members will be as follows:

$$
\begin{aligned}
& \mathrm{P} 3=\frac{\mathrm{A}}{(3-1)+1 / 2} \\
& \mathrm{P} 4=\frac{\mathrm{A}}{(4-1)+1 / 2}
\end{aligned}
$$

The multipliers to be applied to the population in order to obtain the priority list for the method of major fractions are given on page 15 of my book, Congressional Apportionment.

In the method of harmonic mean the difference between the representation of two states is the smallest possible when measured by the absolute difference in the average population per district. The priority list is prepared by dividing the popula-

The Reapportionment of Congress, 45 AM. PoL. Sar. Rev. 153 (I951). The committee consisted of Arthur N. Holcombe, chairman, Zechariah Chafee, Bertram M. Gross, John W. Lederle, Edward H. Litchfield, James K. Pollock, Robert S. Rankin, Laurence F. Schmeckebier, and Walter F. Willcox. 
tion of any state by the harmonic mean of its next higher assignment and of its present assignment, the harmonic mean being a method of averaging absolute differences. The harmonic mean of two numbers (a) and (b) is twice their product divided by their sum, and may be expressed as follows:

$$
\mathrm{H}=\frac{2(\mathrm{a} \times \mathrm{b})}{\mathrm{a}+\mathrm{b}}
$$

Thus the harmonic mean of 2 and 3 is

$$
\mathrm{H}=\frac{2(2 \times 3)}{2+3}=\frac{12}{5}=2 \frac{2}{5}
$$

The multiplier for preparing the priority list number is obtained as follows:

$\mathrm{P}$ (priority Population of any state (A)

list figure) $=\overline{\text { Harmonic mean of its next higher assignment }(\mathrm{k}) \text { and of its }}$ present assignment $(k-1)$

$$
=\frac{\mathrm{A}}{\left(\frac{2(\mathrm{k}-1) \mathrm{k}}{(\mathrm{k}-1)+\mathrm{k}}\right)}
$$

As one representative has already been assigned, the formula for the second member would be as follows:

$$
\begin{aligned}
\mathrm{P} 2 & =\frac{\mathrm{A}}{\left(\frac{2(2-1) 2}{(2-1)+2}\right)} \\
& =\frac{\mathrm{A}}{\left(\frac{2 \times 2}{1+2}\right)} \\
& =\frac{\mathrm{A}}{4 / 3} \\
& =\mathrm{A} \times 3 / 4 \\
& =\mathrm{A} \times 0.75
\end{aligned}
$$

For each succeeding priority list number, $k$ is increased by one, the formulae for the third and fourth numbers being as follows:

$$
\begin{aligned}
& \mathrm{P} 3=\frac{\mathrm{A}}{\left(\frac{2(3-1) 3}{(3-1)+3}\right)} \\
& \mathrm{P} 4=\frac{\mathrm{A}}{\left(\frac{2(4-1) 4}{(4-1)+4}\right)}
\end{aligned}
$$

The multipliers to be applied to the population in order to obtain the priority list for the method of harmonic mean are given on page 35 of my book, Congressional Apportionment.

The method of smallest divisors most nearly equalizes the representative surplus 
of each state-that is, the absolute difference between the representation of an overrepresented state and the number of representatives of an under-represented state multiplied by the population of the over-represented state divided by the population of the under-represented state. A state is over-represented with respect to another state if it has a smaller average population per district; it is under-represented if it has a larger average population per district.

The absolute representation surplus may be expressed as the absolute difference between $\mathrm{a}$ and $\mathrm{b}$ multiplied by $\mathrm{A} / \mathrm{B}$ in which the letters represent the following:

$A=$ Population of over-represented state

$B=$ Population of under-represented state

a $=$ Number of representatives assigned to state $A$

$\mathrm{b}=$ Number of representatives assigned to state $B$.

The priority list number is determined as follows:

$$
\begin{aligned}
\mathrm{P}(\text { priority } & =\frac{\text { Population of any state }(\mathrm{A})}{\text { list number) }} \\
& =\frac{\mathrm{A}}{\mathrm{k}-1}
\end{aligned}
$$

As one representative has been assigned to each state, the formula for the second member would be as follows:

$$
\begin{aligned}
\mathrm{P} 2 & =\frac{\mathrm{A}}{2-1} \\
& =\frac{\mathrm{A}}{1} \\
& =\mathrm{A} \times \text { reciprocal of } 1 \\
& =\mathrm{A} \times 1
\end{aligned}
$$

For each additional priority list number, $\mathrm{k}$ is increased by one.

The multipliers to be applied to the population to obtain the priority list for the method of smallest divisors are given on page 42 of my book, Congressional Apportionment.

In the method of greatest divisors the difference between any two states is the smallest possible when measured by what is termed the absolute representation deficiency, which is the difference between the number of representatives of an under-represented state and the number of representatives of the over-represented state miltiplied by the population of the under-represented state divided by the population of the over-represented state. For the purpose of this method a state is over-represented with respect to another state if it has a smaller average population per district; it is under-represented with respect to another state if it has a larger average population per district.

The absolute representation deficiency may be expressed as the absolute difference between $b$ and $a(B / A)$ in which the letters represent the following: 
$A=$ Population of over-represented state

$\mathrm{B}=$ Population of under-represented state

$\mathrm{a}=$ Number of representatives assigned to state $A$

$\mathrm{b}=$ Number of representatives assigned to state $B$.

The figures in the priority list are obtained by dividing the population by successive numbers of representatives, beginning with its present assignment plus one. The equation is as follows:

$$
\begin{aligned}
\begin{aligned}
\mathrm{P}(\text { priority } \\
\text { list figure })
\end{aligned} & =\frac{\text { Population of any state }(\mathrm{A})}{\text { Its present assignment plus } 1(\mathrm{k}+1)} \\
& =\frac{\mathrm{A}}{\mathrm{k}+1}
\end{aligned}
$$

As one representative has been assigned to each state, the priority number of the second representative would be

$$
\begin{aligned}
\mathrm{P} 2 & =\frac{\mathrm{A}}{1+1} \\
& =\frac{\mathrm{A}}{2} \\
& =\mathrm{A} \times \text { reciprocal of } 2 \\
& =\mathrm{A} \times 0.5
\end{aligned}
$$

For each successive priority list number, $\mathrm{k}$ is increased by one.

The numbers to be applied to the population to obtain the priority list for the method of greatest divisors are given on page $5^{\mathrm{I}}$ of my book, Congressional Apportionment. 